\title{
TOPOLOGICAL ASPECTS OF EPISTEMOLOGY AND METAPHYSICS
}

\section{THOMAS MORMANN}

Abstract. The aim of this paper is to show that (elementary) topology may be useful for dealing with problems of epistemology and metaphysics. More precisely, I want to show that the introduction of topological structures may elucidate the role of the spatial structures (in a broad sense) that underly logic and cognition. In some detail I'll deal with "Cassirer's problem" that may be characterized as an early forrunner of Goodman's "grue-bleen" problem. On a larger scale, topology turns out to be useful in elaborating the approach of conceptual spaces that in the last twenty years or so has found quite a few applications in cognitive science, psychology, and linguistics. In particular, topology may help distinguish "natural" from "not-so-natural" concepts. This classical problem that up to now has withstood all efforts to solve (or dissolve) it by purely logical methods. Finally, in order to show that a topological perspective may also offer a fresh look on classical metaphysical problems, it is shown that Leibniz's famous principle of the identity of indiscernibles is closely related to some well-known topological separation axioms. More precisely, the topological perspective gives rise in a natural way to some novel variations of Leibniz's principle.

0. Introduction: Traditionally, logic is considered as one of the essential pillars of philosophy. Both epistemology and metaphysics are considered as closely related to logic: Epistemology can be conceived of as a sort of applied logic that is engaged in describing the basic laws of (correct scientific) thought, and metaphysics as investigating the most basic structures of the world thereby providing a kind of logic of reality.

In modern times, the traditional basic role of logic for epistemology and metaphysics has become more and more doubtful. Logic in the traditional sense has lost its 
fundamental role for both areas. Even if Feyerabend's notorious "Anything goes" is an untenable exaggeration, it has turned out that traditional logic covers only a limited aspect of the multi-facetted cognitive endevours of modern epistemology and metaphysics. Logic no longer can be considered as a fundamental and unproblematic ingredient of human cognition that can be taken simply for granted. With respect to the areas of cognitive science and epistemology Alberto Peruzzi described this new situation as follows:

The structure of thought is classically described in logical terms. But the roots of logical structure lie deeper than mere symbolic manipulation, for logic inherits meaning from patterns of bodily interaction that involve essential correlations of space and quantity (hence, of geometry and algebra). (Peruzzi (2000, 169/170)).

It goes without saying that the investigation of the "origins" and the "meaning" of logic are questions that cannot be treated exhaustively in a short paper. In this paper I'd like to address some aspects of these questions in a very elementary manner.

1. What are the roots of logical structure and cognition, which lie "deeper than mere symbolic manipulation?

2. What does it mean that logic "inherits meaning" from patterns of bodily interaction that involve space and quantity?

My answer to these questions, then, in a nutshell, is the following (cf. Lakoff 1982):

The roots of logic and cognition lie in our activities as living beings that have to cope with the various challenges of a spatio-temporally structured material world. The spatial-temporal structure of the world is described by some kind of generalized geometry, geometry understood here in a broad as topology as theory of spatial structures in general.

For any classical acount of geometry such a project of a geometrical formulation of logic, cognition, and metaphysics would be a hopeless endevour. What is needed is a 
"geometry" that goes far beyond the confines of traditional (Euclidean) geometry. Fortunately, in the last 150 years or so modern mathematics has provided the conceptual means for such a comprehensive theory of generalized geometry aka topology.

While Euclidean geometry has served as source of inspiration for traditional philosophy over the centuries, in contrast, topology as its modern successor can hardly claim to play an analogous role for contemporary philosophy. How ever successful and fruitful topology may have been in the realm of mathematics and other sciences (empirical science and computer sciences), according to most philosophers, topology is to be considered just as highly sophisticated mathematical theory without philosophical relevance. This state of affairs can hardly be considered as optimal. Fortunately, there is evidence that it may be overcome in the future. In cognitive science, linguistics, and related disciplines modern mathematics has established itself in a way that might help philosophers understand that the ideas of modern mathematics can indeed be philosophically relevant and fruitful. The present paper may be considered as a modest contribution to this endevour. More precisely, the aim of this paper is to show that topology may play a role in elucidating the conceptual roots of the logics and its role in epistemology and metaphysics. $^{2}$

The outline of the paper is as follows. In the next section 2 the philosopher Ernst Cassirer and the psychologist Kurt Lewin are briefly presented as two precursors of a

\footnotetext{
${ }^{1}$ Among the protagonists who played a leading role in this endeavor one may mention Poincaré, Hausdorff, and Kuratowski.

${ }^{2}$ In this endeavor I want to be as accessible as possible. That is to say, often things could be formulated in a mathematically or logically more elegant and more general way (cf. Peruzzi (2000) or Petitot (1995). I prefer to express myself in a much more pedestrian way in order to show that the topological way of doing philosophy (at least in its beginnings) does not depend on a heavy formal apparatus.
} 
kind of topological epistemology avant la lettre. This is interesting not only in itself but also as evidence that analytic philosophy cannot claim to have a monopoly on an affinity to modern mathematics and science - Cassirer and Lewin have been scholars with strong roots in "continental" philosophy. In section 3 we deal with the first highly non-trivial relation of logic and topology that was presented in the 1930s by the mathematician Marshall H. Stone, namely, Stone's Representation Theorem (cf. Stone $(1936$, 1937)). Stone's achievement, which according to many mathematicians is to be considered as a trail-blazing result of 20th century mathematics, shows that the relation between logic and topology is much more than just a nice metaphorical relation but touches the very essence of these disciplines. In section 4 the modern theory of conceptual spaces, inaugurated in the 1990s by Peter Gärdenfors and other scholars is reconsidered from a topological point of view. As an elementary example that topological considerations may be fruitfully applied in the realm of metaphysics, in section 5 topological separation axioms are used to define a variety of novel versions of Leibniz's classical Principle of the Identity of Indiscernibles (LPI).

1. Cassirer's Problem: Begriffsbildung beyond Traditional Logic. The necessity of going beyond the traditional logic as the art of correctly manipulating logical symbols was not a problem perceived solely in the context of analytical philosophy. This problem arose well before analytical philosophy proper came into being. Evidence for this is Ernst Cassirer's Neo-kantian philosophy of science. Cassirer's philosophy of science was concept-oriented par excellence:

The theory of the concept becomes a cardinal problem of systematic 
philosophy. It becomes the nub around which logic, epistemology, philosophy of language and cognitive psychology are rotating (Cassirer $1928,163)$.

Already in his first systematic opus magnum Substance and Function (1910) Cassirer had conceived philosophy of science as a theory of the formation of scientific concepts. His theory was empirical in the sense that philosophy should not decree what scientific concepts were and how they worked from the philosophical armchair, so to speak. Rather, since scientific concepts evolved in the historical evolution of science, it was the task of philosophy of science to study this conceptual development of science and to make philosophical sense of it, not to legislate it according to some preconceived philosophical ideas. He considered traditional logic, as a part of traditional philosophy, as epistemologically deficient:

Traditional logic comprises, so to speak, with the same love "valid" and "unvalid", "useful" and "non-useful" concepts. Herein resides, ... seen from an epistemological point of view ... its decisive fault. Hence now it becomes evident that a concept may possess its complete formal "correctness" in the sense of classical logical theory, that it may be construed quite "correctly" without telling us anything about its specific epistemological usefulness (Cassirer 1928, 132).

Already 20 years earlier in the introduction to Substance and Function he mentions the following drastic example that can be traced back to the 19th century logician and philosopher Hermann Lotze: ${ }^{3}$

${ }^{3}$ In Anglo-Saxon analytic philosophy, Nelson Goodman and Carl Gustav Hempel are said to have been the first philosophers who put the issue of distinguishing between "valid" and "non-valid" concepts on the agenda. As is well-known, this has lead to a whole "industry" that is engaged in finding criteria for distinguishing between "non-valid" predicates such as "grue" and "bleen" on the on side and entrenched or projectible ones as "blue" and "green" on the other side. Hempelian paradoxes of confirmation have found hardly less attention. Formulated in a very 
If we group cherries and meat together under the attributes red, juicy and edible, we do not thereby attain a valid logical concept but a meaningless combinations of words, quite useless for the comprehension of the particular cases. Thus it becomes clear that the general formal rule in itself does not suffice; that on the contrary, there is always tacit reference to another intellectual criterion to supplement it. (Cassirer (1910 (1923), 7).

As Cassirer points out, in Aristotle's philosophy this other supplementary criterion is that the gaps, which are left in logic, are filled in and made good by metaphysics. For Aristotle a concept is not an arbitrary group of things. The selection of what is common remains an empty play of ideas if it is not assumed that what is thus gained is, at the same time, the real Form which guarantees the causal and teleological connection of particular things. For Aristotle, according to Cassirer:

The process of comparing things and of grouping them together accodring to similar properties ... does not lead to what is indefinite, but if rightly conducted, ends in the discovery of the real essences of things. (Cassirer (1910 (1923), ibid.))

This close Aristotelian connection between logic and metaphysics has disappeared in modern times. We have to rely on other, not always well-understood means to distinguish honest concepts from meaningless, but formally correct conceptual chimeras. In our daily practice of life and science, we are hardly ever confronted with Cassirer's (or Goodman's or Hempel's) problem. We seem to have an innate intuition of which concepts are natural and which are not. Philosophy cannot remain

simplistic way they tackle the problem why a green frog cannot count as a confirmation of the "law" that "All ravens are black" although a green frog may considered as a confirmation of the logically equivalent "law" that "All non-black things are no-ravens. 
content with this intuitive state of affairs. It remains an important task of epistemology and philosophy of science to explicate how this is achieved. The aim of this paper is to tackle this problem by using geometrical and, more generally, topological methods that may be conceived as a kind of relativized or historized $a$ priori.

As a first step in the task of coming to terms with Cassirer's problem it is expedient to formulate Cassirer's problem in a more precise manner by interpreting concepts extensionally. This means that we identify a concept A with its extension (conceived as a subset of a universal set $U$ ) such that the concept A is represented by the set of objects that instantiate it. Then, Cassirer's problem can be informally expressed in the following way: There are many more subsets $A \subseteq U$ than natural concepts (predicates, properties). Set theory does not distinguish between "good" sets, which could represent "natural" concepts, and "bad" sets that do not. It does not offer means to overcome this shortcoming by itself. Nevertheless, it gives some useful hints. The deficiencies of a purely set-theoretical modeling of concepts can be overcome if the unstructured base sets $U$ of traditional extensional logics are replaced by structured conceptual spaces: Then the basic thesis of a geometrical or topological epistemology can be formulated as follows:

Natural concepts correspond to well-formed, subsets of a conceptual spaces. Non-natural concepts are represented by geometrically non-well-formed sets.

Such a geometrical epistemology is partially an empirical theory: we do not know a priori which geometric structures of conceptual spaces are appropriate for the characterization of natural concepts of a domain. We have to find out this through the empirical investigation of examples. 
Let us consider the following well-known example: The simplest model for a conceptual space of color predicates ("red", "blue", "yellow", ...) is the well-known "color circle" or, a bit more advanced, the "color spindle" that represents basic color predicates such as "red" or "blue" by certain areas or regions of these spaces (cf. Gärdenfors $(2000,2014))$. Due to the geometrical and topological structure of the circle, this representation renders plausible that disjunctive color predicates such as "red or green" fail to be natural predicates. This is confirmed by empirical investigations according to which there are no natural languages that possess simple color terms for expressing disjunctive color properties. In other words, there is a correspondence between "intuitive naturalness" and "structural wellformedness", since connected areas of the circle are geometrically better-behaved than scattered and disconnected one.

From this example one may conjecture that a necessary condition for color predicates to be natural is that they can be represented by connected subsets of the color circle. As will be argued in more detail later, being represented by connected areas of the representing space is only one requirement among others that "good" concepts have to satisfy. It is a non-trivial problem to find other useful criteria.

Regardless of the details of how this can be achieved, already here it is expedient to warn against a possible fundamental misunderstanding concerning the epistemological status of the spatial structure of conceptual spaces. It would be quite misleading to conceive them as "mere metaphorical talk" or just as "pictorial illustrations" ("Veranschaulichungen”) without cognitive significance.

As an early witness for this thesis, I'd like to mention the psychologist and philosopher Kurt Lewin, who may be considered as the first scientist who ever 
envisaged a deep non-trivial relation between topology and cognitive psychology. ${ }^{4}$ Since Lewin may not be so well-known among philosophers, the following succinct biographical remarks may be in order. Lewin was a former student of Cassirer, later he belonged to the Berlin group of philosophers and scientists that gathered around Reichenbach and may be considered as an ally of the Vienna Circle in their common aim to render philosophy a scientifically respectable entreprise. In the Introduction to his book The Principles of Topological Psychology (Lewin 1936) he wrote:

The Principles of Topological Psychology is the result of a very slow growth. I remember the moment when - more than ten years ago - it occurred to me that the figures on the blackboard which were to illustrate some problems for a group in psychology might after all be not merely illustrations but representations of real concepts. ... Knowing something of the general theory of point sets, I felt vaguely that the young mathematial discipline of "topology" might be of some help in making psychology a real science. (Lewin $(1936$, vii))

Lewin's The Principles of Topological Psychology may be seen as the historically first attempt to apply topological concepts to problems of cognitive psychology. I don't want to enter into a detailed discussion of Lewin's book. The important point is to take serious Lewin's thesis according to which the concepts of psychology may be usefully represented spatially:

... that the figures on the blackboard might be not merely illustrations but representations of real concepts. I had already ... in 1912 defined the thesis ... that psychology, dealing with manifolds of coexisting fact, would be finally forced to use not only the concept of time but that of space too. (ibidem)

\footnotetext{
${ }^{4}$ For some interesting remarks on Lewin's “topological field semantics" see Wildgen (2000, 216-217) and Wildgen (2001).
} 
That is to say, for Lewin - and this renders him a precursor of modern geometrical or topological accounts of cognitive science and epistemology - the phenomena of logic and cognition can be fruitfully represented by geometrical and topological concepts. Sure, Lewin's “topological psychology" uses only quite elementary and intuitive topological concepts. A stubborn despiser of a topological perspective may still dismiss it as merely metaphorical. In order to definitively refute the prejudice that topology has nothing more to offer than mere metaphorical illustrations for logical phenomena. In order to refute this claim, in the next section I'd like to briefly discuss Stone's representation theorem in some more detail. Stone's theorem is to be considered as the first deep connection between logic and topology. It establishes a profound relation between logic and topology that up to now has only insufficiently been eploited philosophically, or so I want to argue. ${ }^{5}$

2. Topological Aspects of Logic: Stone's Representation theorem. Since the advent of Tarskian semantics the set-theoretical representation of predicates and relations has become a standard method in logic. An innate weakness of settheoretical semantics is that it ignores "Cassirer's problem", i.e., predicates may have any extensions whatsoever, a distinction between "good" and "not-so-good" predicates according to the well-formedness of their extensions cannot be not drawn - all subsets A of a semantic universe $U$ are equally admissible. Since some time quite a few authors are engaged in the task of refining the set-theoretical approach by amending set-theory by topological methods. For instance, if one is interested in distinguishing vague concepts from precise concepts, topology may come to the

\footnotetext{
${ }^{5}$ A modern presentation of its mathematical content may be found in Mac Lane and Moerdijk (1992).
} 
rescue in a quite natural way. Instead on a set-theoretical universe one relies on an appropiate topological space. Then vague predicates A may be characterized by the property that all objects $\mathrm{x}$ that instantiate $\mathrm{A}$ do this in a stable way, i.e., any sufficiently small variation $\mathrm{x}$ ' of an $\mathrm{x}$ that instantiates A still instantiates A. To make this precise a topological structure is required. A recent, very interesting elaboration of this topological refinement of classical set-theoretical semantics can be found in Ian Rumfitt's The Boundary Stones of Logic (Rumfitt 2015). In this work, Rumfitt offers (among many other things) a topological account of conceptual spaces that sheds interesting new light on the role of prototypes for the topological structure of conceptual spaces. ${ }^{6}$

This shows that topological structures (and possibly other structures as well) may be used to refine traditional logic in such a way that Cassirer's problem can be tackled. In a nutshell, this procedure can be characterized as a structural amendment of the classical approach. It is based on the idea that appropriate relational systems such as topological spaces provide information that can be exploited in some way or other to distinguish between natural and not-so-natural concepts.

Topology, however, may not only be used to refine classical logic by distinguishing, for instance, between vague and precise concepts. Topology is also helpful for investigating and elucidating the topic of Boolean ${ }^{7}$ algebra itself. This is evidence that topology is a kind of general logic of space, is more fundamental than logic as

\footnotetext{
${ }^{6}$ More precisely, relying on a prototype account of concepts he shows that prototypes can be used to define a topological structure on conceptual spaces that can distinguish between "good" ("natural") concepts and "not-so-good" ("non-natural") concepts. This prototype topology is very different from familiar topologies such as the topology of Euclidean spaces.

${ }^{7}$ Actually, this is not true only for Boolean logic but for other logics as well. Incidentally, the case of Boolean logic is only the historically first one. Stone's theorem may be considered as a source of a flourishing quite general discipline of "spatial logics" documented, for instance, in the monumental Handbook of Spatial Logics (2007) edited by Aiello, Pratt-Hartmann and van Benthem.
} 
understood traditionally as a purely symbolic discipline quite unrelated to any spatial considerations. It is the achievement of Marshall Stone to have set the foundations for a topological reformulation of logic that turned to be extremally fruitful in the following decades of the 20th century till today (cf. Mac Lane and Moerdijk (1992)). The modest aim of this paper is only to provide a superficial look on the most elementary aspects of this new perspective onto logic. In a nutshell Stone's great achievement is to have constructed for each Boolean algebra B a topological space St(B) in an essentially 1-1-way such that the conceptual (algebraic) devices of the algebra B could be used to shed new light on the topology of $\mathrm{St}(\mathrm{B})$ and viceversa, i.e., the conceptual (topological) devices of the topological space $\mathrm{St}(\mathrm{B})$ offered to insights into the algebraic (logical) structure of B. More precisely, Stone showed that for each Boolean algebra B one can construct a topological space $\mathrm{St}(\mathrm{B})$ :

(1) The points of the Stone space $\mathrm{St}(\mathrm{B})$ of B can be constructed as the maximal filters (ultrafilters) of the Boolean algebra B. As has been shown, this construction has may applications in lattice theory, order theory, and algebra in general. As is well-known, without the assumption of the axiom of choice or some similar principle the existence cannot be ensured. Hence the construction of a Stone space $\mathrm{St}(\mathrm{B})$ may be characterized as a conceptually very demanding endevour.

(2) The set $\mathrm{St}(\mathrm{B})$ of ultrafilters of B is topologized in a canonical way, such that in the topological space $\operatorname{St}(B)$ the the elements of B can be identified with very special genuine topological structures of $\operatorname{St}(B)$, namely, with clopen sets, i.e., sets that are open and closed with respect to the canonical topology of the space $\mathrm{St}(\mathrm{B})$.

The spaces that are treated in traditional topology such as the Euclidean spaces E only have very few clopen regions, namely, the empty set $\varnothing$ and the entire space E itself. Thus, the topologization of Boolean logic carried out by Stone is very far away 
from any intuitive and merely "metaphorical" geometrical reformulation of logic. There is no need to go into the details here. Be it sufficient to say that the Stone space $\mathrm{St}(\mathrm{B})$ of a non-trivial Boolean algebra $\mathrm{B}$ is a rather strange space in the sense that its topology has many clopen elements. In a nutshell, then, Stone's representation theorem of Boolean logic may be encapsulated in the formula

$$
\mathrm{B}=\operatorname{Clopen}(\operatorname{St}(\mathrm{B}))
$$

Here $\mathrm{B}$ is any Boolean algebra, $\mathrm{St}(\mathrm{B})$ the Stone space of $\mathrm{B}$, and Clopen( $\mathrm{St}(\mathrm{B}))$ the set of clopen sets of the topological space $\mathrm{St}(\mathrm{B})$.

In the decades after Stone had formulated his theorem, it found many applications and generalizations in a variety of mathematical disciplines (cf. Johnstone 1982). This is not the place to deal with this issue in any detail. Just one remark. At first view, one might have doubted the topological relevance of Stone's theorem, since Stone spaces are very different from the topological spaces that traditional topology has dealt with. This impression is quite misleading. A generalization of Stone's theorem establishes that for many "ordinary" topological spaces an analogous theorem can be proved: If $\mathrm{X}$ is a reasonably well-behaved topological space, for instance, a Euclidean space, then the lattice OX of open sets is a complete Heyting algebra. Moreover, any well-behaved Heyting algebra $\mathrm{H}$ uniquely defines a topological space $\mathrm{pt}(\mathrm{H})$ such that the lattice of open sets $\mathrm{O}(\mathrm{pt}(\mathrm{H}))$ is isomorphic to $H$. That is, one obtains the analogous correlation for Heyting algebras and general topological spaces as the one that Stone proved for Boolean algebras and Stone spaces.

Stone showed that topology was not just a mathematical discipline among others, but rather a method or a conceptual organon to be applied fruitfully in many areas of mathematics that at first view seemed to be quite unrelated to topology proper. This 
led him to formulate what became famous as "Stone's maxim", namely, the dictum that, being confronted with any mathematical problem whatsoever, "one must always topologize", i.e., one must always attempt to conceive it as a topological problem such that its solution could be achieved by topological means.

Taking into account that logic, as one of the corner stones of philosophy, has its roots in topology, topology understood as general theory of spatial structures, it does not seem unreasonable to generalize Stone's maxim to at least some areas of philosophy as well: Given a philosophical problem, say, of logic or metaphysics, one should try to exhibit its topological (i.e. spatial) aspects in order to apply topologically inspired methods to solve it. If this general strategy should turn out to be feasible, formal philosophy, which has been traditionally dominated by logical problems and logical methods for more than one century, would give way to a kind of topological philosophy. In certain area this shift is already under way. As an example one may mention epistemology for which the theory of conceptual spaces plays an ever more important role.

To be sure, in order that a geometrical or topological epistemology can get off the ground, a sufficiently general notion of space is necessary. We should be prepared to accept a topology of logic, and, more generally, a topology of thought that uses conceptual spaces whose topology differs considerably from the familiar topology of Euclidean space we are accostumed to.

3. Conceptual Spaces as a Framework for Topological Epistemology. Over the last two decades or so conceptual spaces as a framework for the geometrical or topological representation of concepts and knowledge has been highly influential. It 
has contributed a lot to elucidate and articulate the spatial roots of our knowledge and cognition. Many researchers in cognitive psychology and cognitive science have contributed to the development of the conceptual spaces approach (even Kurt Lewin may be considered as an early precursor). Nevertheless, this approach gained real momentum only in the last decade of the 20th century with the work of Peter Gärdenfors (cf. Gärdenfors (1996, 2000, 2014)).

The basic technical idea of this approach is that concepts can be usefully represented geometrically or topologically as regions of well-defined geometrical structures, socalled conceptual spaces. More precisely, according to Gärdenfors, concepts can be represented by convex regions of conceptual spaces that result from Voronoi tessellations that are defined with the help of the prototypes of these concepts. More generally, meanings can be represented by geometrical structures of conceptual spaces (cf. Gärdenfors (2014)).

This geometrical representability is no coincidence. Rather, our conceptual systems are constructed by us, who are creatures that live in a spatio-temporally structured material world. Thus,

[T]hese conceptual systems grow out of our bodily experience and make sense in terms it; moreover, the core of our conceptual systems is directly grounded in perception, body movement, and experience of a physical and social character. (Lakoff 1987, xiv)

For all experiences whatsoever (perhaps apart from mystical experiences) their spatial character is essential. That means in order to be meaningful and communicable they have to be conceived as being located in a conceptual space that is metaphorically related to physical physical space in some way or other. This is - in very general terms - the content of Lakoff's Spatialization of Form Hypothesis" (Lakoff 1987, p. 283) that lies at the heart of his Cognitive Semantics approach 
whose explicit aim is to elaborate a theory of embodied cognition that conceives all our cognitive endeavours - even the most abstract ones such as mathematics - as determined by the contingencies of our existence as natural beings in a material spatio-temporal world .

According to cognitive semantics, conceptual structures depend on the perceptions and actions of the cognizing subjects. This entails that these structures reflect in some way or other the geometrical ( = topological) structure the spatio-temporal world those subjects live. As Lakoff convincingly argued, this entails that cognitive structures are not propositional, but image-schematic. This means, ultimately, that they depend on the topological structures of the spatio-temporal material world we belong to.

The regions of conceptual spaces that correspond to concepts are constituted by the sets of points that represent those objects, processes, or events which exhibit the key perceptual properties associated to each considered region.

Every conceptual space is endowed with a metric that measures the similarity between objects. For any two objects, events, processes, or whatever the elements of the conceptual space are assumed to represent, $\mathrm{x}$ is the more similar to $\mathrm{y}$ the closer it is to y according to the metric. This notion of similarity is a similarity-in-a-givenrespect, that is encapsulated in the specific metrical structure of a space - not an overall similarity. It may well be the case that some aspects of the objects to be compared are ignored by that metrical structure.

One of the best known and most familiar example of a conceptual space is the threedimensional Euclidean space which serves to represent proximity relations between physical objects. Other examples of conceptual spaces are the spaces of temporal, auditory, olfactory and taste experiences. For the latter ones it may be not clear that 
they can be endowed with a fully-fledged Euclidean structure. Rather, they are endowed only with a simpler topological structure of some kind.

In Gärdenfors's conceptual spaces the categorization of an object $g$ under a particular concept is the result of a process that (i) evaluates the distances of $g$ from the prototypes of all the relevant concepts within the con sidered context, and (ii) classifies an object $\mathrm{g}$ under the concept $\mathrm{C}$ whose prototype $\mathrm{p}_{\mathrm{C}}$ is the most similar to g. If it happens that there are several different prototypes $p_{1}, \ldots, p_{n}$ maximally close to $\mathrm{g}$, then $\mathrm{g}$ is located on the boundary of the cells defined by the prototypes $\mathrm{p}_{1}, \ldots$, $\mathrm{p}_{\mathrm{n}}$ (for details cf. Rumfitt (2015)).

Cassirer's problem of how natural concepts can be distinguished from gerrymandered "Goodmanian" concepts shows up in the context of conceptual spaces in a quite natural manner: As said, concepts are identified with specific wellformed spatial regions. For instance, the concepts "blue" and "green" correspond to well-formed regions of the color space. In contrast, the Goodmanian counterparts "grue" and "bleen" turn out to be as represented by non-well-formed or at least less well-formed regions of the color space (cf. Gärdenfors (2000, chapter 3), Mormann (1993, 231). This is indeed the case. Thus, due to the geometrical structure, not all subsets of a conceptual space correspond to natural concepts.

Due to the selective force of the topological structure not all subsets of a conceptual space C correspond to natural concepts. However, Gärdenfors does not give necessary and sufficient conditions that a region represents a natural concept. Instead, he only proposes a necessary condition for naturalness, to wit, convexity. A subset A of an appropriate (metrical) space $\mathrm{C}$ is convex iff for any pair of points $\mathrm{x}, \mathrm{y}$ of A the line segment connecting $\mathrm{x}$ and $\mathrm{y}$ lies, in its entirety, in A as well.

Then, according to Gärdenfors, a "good" concept is to be represented by a convex 
region of a conceptual space. For the following it is sufficient to consider some elementary examples. Let us assume that the conceptual space $\mathrm{C}$ has the structure of an Euclidean space (or some derivative of it, e.g., a locally Euclidean manifold such as the well-known color spindle, (cf. Gärdenfors (2014, p.23), (Gärdenforst (1990)). Then, convex regions as representatives of natural properties in conceptual spaces may be defined as follows:

(3. 1) Definition ("Criterion C"). A region R is convex iff for any two points $\mathrm{x}$ and $\mathrm{y}$ in $\mathrm{R}$, all points between $\mathrm{x}$ and $\mathrm{y}$ are also in $\mathrm{R}$. A natural property is represented by convex region of a conceptual space D.

Gärdenfors' motivation for the convexity requirement invokes the idea of learnability: if some objects located at $\mathrm{x}$ and $\mathrm{z}$ are both instantiations of a "convex" concept $\mathrm{C}$, then any object $\mathrm{y}$ that is located "between" $\mathrm{x}$ and $\mathrm{z}$ will also instantiate the concept C. This suggests that for any object y that is located in this way, it is easily learned that it also instantiates $\mathrm{C}$ - provided one already knows that $\mathrm{x}$ and $\mathrm{z}$ are instantiations of C. As Gärdenfors pointed out more than once, a plausible theory of concepts should explain somehow that a conceptual system (i.e., a system of concepts) is learnable by finite creatures possessing only a limited capacity of memory. "Criterion C" is intended to cope with this challenge.

"Learnability" is not the only adequacy condition that conceptual systems have to satisfy if they strive to be considered as adequate. Recently, Douven and Gärdenfors have proposed a list of "design principles (for conceptual systems based on conceptual spaces with prototypes)" that good conceptual systems should satisfy (Douven and Gärdenfors (2018)):

(3.2) Definition ("Design Principles for Conceptual Systems”). 
(1) Parsimony: The conceptual structure must not overload the system's memory.

(2) Informativeness: The concepts should be informative, meaning that they should jointly offer a good and roughly equal coverage of the domain of classification cases.

(3) Representation: The conceptual structure should be such that it allows the system to choose for each concept a prototype that is a good representative of all items falling under the concept.

(4) Contrast: The conceptual structure should be such that prototypes of different concepts can be so chosen that they are easy to tell apart.

(5) Learnability: The conceptual structure should be learnable, ideally from a small number of instances.

To be sure, the principles (1) - (5) may not always single out a unique system among several rival ones, but at least they help narrow down the number of competitors. This is the best one can hope for. After all, the strict connection between metaphysics and epistemology seems to be once and for all lost, conceptual systems remain forever more or less corroborated hypotheses that have to be assessed by appropriate pragmatic criteria. The general design criteria proposed by Douven and Gärdenfors may be taken as general guidelines for coming to terms with this task, not as foolproof recipes that guarantee fully satisfying results.

\section{Topological Metaphysics: Separation Axioms as Leibnizian Principles of the}

Identity. The aim of this section is to show that topology may be a useful tool to elucidate a classical metaphysical principle in a new way, namely, Leibniz's 
Principle of the Identity of Indiscernibles. More precisely, it will be shown that from a topological perspective Leibniz's original principle is only one member of a large family of similar principles that all can be conceived as topological separation axioms

Leibniz's Principle of the Identity of Indiscernibles (hereafter called LPI) is usually formulated as follows: If, for every property $F$, object $x$ has $F$ if and only if object $y$ has $F$, then $x$ is identical to $y$. In symbolic notation:

(4.1) (Leibniz's Principle of the Identity of Indiscernibles). Let F be a property, and $\mathrm{x}, \mathrm{y}$ be objects. Then Leibniz's Principle of the Identity of Indiscernibles holds if and only if the following implication holds:

$$
\forall \mathrm{x}, \mathrm{y} \forall F(F x \leftrightarrow F y) \rightarrow x=y .
$$

(Stanford Encyclopedia, Forrest (2016))

The logically equivalent transposition of (LPI) is sometimes called the Principle of Dissimilarity of the Diverse (PDD), namely: If $x$ and $y$ are distinct objects, then there is at least one property $\mathrm{F}$ that $x$ has and $y$ does not, or that $\mathrm{y}$ has and $\mathrm{x}$ does not have. In symbolic notation:

(4.2) The Principle of the Dissimilarity of the Diverse.

$$
\forall \mathrm{x}, \mathrm{y}(\mathrm{x} \neq \mathrm{y} \rightarrow \exists \mathrm{F}((\mathrm{Fx} \text { and not } \mathrm{Fy}) \text { or }(\mathrm{Fy} \text { and not } \mathrm{Fx})))
$$

(Stanford Encyclopedia, Forrest (2016)).

The status of LPI is controversial, to put it mildly. According to some, it is simply false, to others, it is trivially true. Still others claim that there are various versions of LPI, some of them true, others false. These diverging contentions indicate that not all 
people understand the same as "Leibniz's principle of the identity of indiscernibles". This is indeed the case. For instance, if one accepts quantification over all properties whatsoever, then no Leibniz principle is needed at all. As Whitehead and Russell put it:

It should be observed that by indiscernibles" [Leibniz] cannot have meant two objects which agree as to all their properties, for one of the properties of $\mathrm{x}$ is to be identical with $\mathrm{x}$, and therefore this property would necessarily belong to $\mathrm{y}$ if $\mathrm{x}$ and $\mathrm{y}$ agreed in all their properties. Some limitation of the common properties necessary to make things indiscernible is therefore implied by the necessity of an axiom. ... [W]e may suppose the common properties required for indiscernibility to be limited to predicates. ... (Whitehead and Russell, Principia Mathematica (Introduction, p. 57))

In other words, Whitehead and Russell exclude "identity properties" such as "being identical with $\mathrm{x}$ " from the domain over which the quantifier of Leibniz's principle runs since such properties as not defined by predicates but predicate functions.

Dealing with Leibniz's principle of the identity of indiscerniable we are confronted with what may be called the „limitation problem“, i.e., the problem of giving criteria how to restrict the domain of properties that have to be taken into account for Leibniz's principle.

How ever the limitation problem is solved, not all properties should be admitted: As just said, if for all objects $\mathrm{x}$ one admits the property „being identical with $x^{\text {“ }}$ Leibniz's principle holds for trivial reasons. Thus, „being identical with $\mathrm{x}^{\text {“ }}$ should not be considered as a "good“ property that has to be taken into account for a reasonable Leibniz's principle. So, in dealing with Leibniz's principle we are confronted again with a version of Cassirer's problem, namely, the distinction between „good“ and „not so good“ properties, i.e., properties that are admitted to 
occur in the range of the principle's quantifier and those that are not. As we shall see, topology may help cope with this problem.

The very key observation here is that the validity of Leibniz's principle is nothing but the assertion that the topological separation axiom $\mathrm{T}_{0}$ holds (cf. Steen and Seebach, Jr. (1978, p.11)). In a nutshell, LPI is just $\mathrm{T}_{0}$ in disguise. More precisely, the following holds:

(1) LPI is equivalent to $T_{0}$ for appropriately defined topological properties.

(2) $\mathrm{T}_{0}$ is the simplest member of a large family of topological separation axioms $\mathrm{T}_{\mathrm{i}}, 0 \leq \mathrm{i}$.

Thus, it is plausible to conjecture that at least some of the higher separation axioms $\mathrm{T}_{\mathrm{i}}(\mathrm{i}>0)$ may give rise to various novel Leibnizian principles that deserve to be studied in metaphysics. The details are as follows. From an extensional point of view we may (pace Wittgenstein) identify a world $\mathrm{X}$ with a set of objects existing in $\mathrm{X}$. Then a property may be identified with a subset $\mathrm{F} \subseteq \mathrm{X}$. In this setting LPI boils down to the assertion that two objects $\mathrm{x}, \mathrm{y} \in \mathrm{X}$ are identical iff they they are elements of the same (admissible) properties $\mathrm{F} \subseteq \mathrm{X}$. Clearly, if any subsets $\mathrm{F} \subseteq \mathrm{X}$ whatsoever are allowed to serve as properties, Leibniz's principle is trivially true. If one wants to avoid this unwelcome result, one has to exclude some unnatural properties in some way or other. In other words, one has to solve Whitehead and Russell's limitation problem, or, to express it in still another way, one has to cope with Cassirer's problem of distinguishing between good and not-so-good properties. Here topology may come to the rescue. The very definition of a topological structure can be interpreted as a response to the limitation problem in the sense that a topological 
structure on a set $\mathrm{X}$ can be used to distinguish topologically well-behaved subsets from non-well-behaved ones. The details are as follows:

(4.3) Definition. Let $\mathrm{X}$ be a set and PX its power set. A topological structure on $\mathrm{X}$ space is a relational structure $(\mathrm{X}, \mathrm{OX})$ with $\mathrm{OX} \subseteq \mathrm{PX}$ satisfying the requirements that $\varnothing, \mathrm{X} \in \mathrm{OX}$, finite intersections and arbitrary unions of elements of $\mathrm{OX}$ are elements of OX. The elements of OX are called open sets, their set-theoretical complements are closed elements of the topological structure (X, OX).

On every set $\mathrm{X}$ there are two extreme topological structures $\left(\mathrm{X}, \mathrm{O}_{0} \mathrm{X}\right)$ and $\left(\mathrm{X}, \mathrm{O}_{1} \mathrm{X}\right)$ defined by $\mathrm{O}_{0} \mathrm{X}:=\{\varnothing, \mathrm{X}\}$ and $\mathrm{O}_{1} \mathrm{X}:=\mathrm{PX}$. With respect to set-theoretical inclusion $\subseteq$ all topological structures $(\mathrm{X}, \mathrm{OX})$ on $\mathrm{X}$ lie between these two extreme topologies: $\mathrm{O}_{0} \mathrm{X} \subseteq \mathrm{OX} \subseteq \mathrm{O}_{1} \mathrm{X}$

Obviously, for a non-trivial set $\mathrm{X}$ there are many different topological structures OX. Thus, the point of topologizing a world $\mathrm{X}$ is not simply to note that a topology on $\mathrm{X}$ exist, but to choose an interesting one.

(4.4) Definition (Topological Properties). Let (X, OX) be a topological space, A topologically admissible property of $\mathrm{x} \in \mathrm{X}$ is an open set $\mathrm{a} \in \mathrm{OX}$ such that $\mathrm{x} \in \mathrm{a}$. The system of admissible properties of $x$ is denoted by $\mathrm{O}(\mathrm{x}):=\{\mathrm{a} ; \mathrm{x} \in \mathrm{a}$ and $\mathrm{a} \in \mathrm{OX}\}$. The Heyting algebra OX is called the topological property system of (X, OX).

After these clarifications we can tackle the problem of determining those topological structures that give rise to interesting Leibnizian principles LPI and those which do not: 
(4.5) Examples (Leibniz's Principle LPI of the Identity of Indiscernibles for

topological property systems.) Let (X, OX) be a topological space.

(1) For the indiscrete topology $\mathrm{O}_{0} \mathrm{X}=\{\varnothing, \mathrm{X}\}$ Leibniz's principle of the identity of indiscernibles is clearly false: According to the property system of $\mathrm{O}_{0} \mathrm{X}$, there is only one non-empty property, namely the trivial property $\mathrm{X}$ that is instantiated by all elements of X. Thus, Leibniz' principle is false for all worlds $\mathrm{X}$ that have more than one element.

(2) For the discrete topology $\mathrm{O}_{1} \mathrm{X}=\mathrm{PX}$, Leibniz's principle of the identity of indiscernibles is trivially true since it contains for every object $\mathrm{x} \in \mathrm{X}$ the identity property "being identical with x", namely, the singleton $\{x\}$. Hence, distinct object $\mathrm{x} \neq \mathrm{y}$ have different properties $\{\mathrm{x}\}$ and $\{\mathrm{y}\}$, and LPI is true.

Admittedly, this is not very exciting. But at least it suggests how the undesired “identity properties" "being identical with x", incriminated already by Russell and Whitehead in their discussion of the Leibniz principle, can be excluded by topological means in a natural way: A necessary condition a „good“ topology OX should satisfy is that it has no isolated points, i.e., $\{x\} \notin \mathrm{OX}$ for all $\mathrm{x} \in \mathrm{X}$. What about intermediate topologies $\mathrm{OX}$ strictly between $\mathrm{O}_{0} \mathrm{X}$ and $\mathrm{O}_{1} \mathrm{X}$ ?

At first look, the problem whether a topological space (X, OX) satisfies the topological Leibniz principle LPI for its canonical property system OX may appear to be a somewhat contrived hybrid of a philosophical a topological problem. Actually, it is not. Rather, this question has attracted considerable interest among topologists since the beginnings of topology more than one hundred years ago. More precisely, we will show in a moment that the validity of LPI is equivalent to the weakest topological separation axiom $\mathrm{T}_{0}$ : 
(4.6) Definition. Let $(X, O X)$ be a topological space, $x, y \in X$, and $a \in O X$. The topological space $(\mathrm{X}, \mathrm{OX})$ is a $\mathrm{T}_{0}$-space iff the for all distinct $\mathrm{x}, \mathrm{y}$ there exists an open set $\mathrm{a} \in \mathrm{OX}$ such that either $\mathrm{x} \in \mathrm{a}$ and $\mathrm{y} \notin \mathrm{a}$, or $\mathrm{x} \notin \mathrm{a}$ and $\mathrm{y} \in \mathrm{a}$.

The axiom $T_{0}$ has often been considerated as a minimal requirement that "good" topological spaces have to satisfy. To obtain interesting specific results, however, one has to assume the validity of certain stronger separation axioms $T_{i}, i>0$. As the starting point for the endeavor of interpreting topological separation axioms as Leibnizian principles of the identity of indiscernibles we begin with the following easily proved proposition:

(4.7) Proposition. A topological space $(X, O X)$ satisfies the separation axiom $T_{0}$ if and only if the topological property system OX satisfies Leibniz's law LPI.

In order to emphasize the intimate relation between $\mathrm{T}_{0}$ and LPI, in the following let us denote LPI by $\mathrm{LPT}_{0}$.

Proposition (4.7) opens the gate for constructing a close connection between topological separation axioms and Leibnizian principles of the identity of indiscernibles:

$\mathrm{T}_{0}$ is just the first member of a large family of separation axioms $\mathrm{T}_{i}, \mathrm{i} \geq 0$. The fact that Leibniz's principle and $\mathrm{T}_{0}$ are equivalent suggests that not only $\mathrm{T}_{0}$ but also other members $T_{i}$ of the family of separation axioms can be interpreted in terms of (generalized) Leibnizian principles of identity of indiscernibles. This is indeed the case as is confirmed by the following few examples (that could be easily multiplied): 
(4.8) Definition. A topological space $(X, O X)$ is a $T_{1}$-space iff for all distinct points $\mathrm{x}, \mathrm{y}$ there exist open sets $\mathrm{a}, \mathrm{b} \in \mathrm{OX}$ such that $\mathrm{x} \in \mathrm{a}$, and $\mathrm{y} \in \mathrm{b}$, such that $\mathrm{x} \notin \mathrm{b}$ and $\mathrm{y} \notin \mathrm{a}$.

For the corresponding Leibnizian principle one obtains:

(4.9) Theorem (LPT 1 -priniciple of the identity of indiscernibles). A system of properties $\mathrm{P}:=\{\mathrm{F} ; \mathrm{F} \in \mathrm{P}\}$ satisfies the $\mathrm{T}_{1}$-Leibnizian principle of the identity of indiscernibles iff for all

$\left(\mathrm{LPT}_{1}\right) \quad \forall \mathrm{x}, \mathrm{y}(\mathrm{x} \neq \mathrm{y} \rightarrow \exists \mathrm{F}((\mathrm{Fx}$ and not $\mathrm{Fy})$ or $\exists \mathrm{G}(\mathrm{Gy}$ and not $\mathrm{Gx})))$

A topological space $(\mathrm{X}, \mathrm{OX})$ is a $\mathrm{T}_{1}$-space iff the topological property system $\mathrm{OX}$ satisfies $\left(\mathrm{LPT}_{1}\right)$.

There is no reason to stop here. One may go on to interpret further separation axioms as principles of the identity of indiscernibles analogously:

(4.10) Definition. The topological space $(\mathrm{X}, \mathrm{OX})$ is a $\mathrm{T}_{2}$-space iff for all distinct points $\mathrm{x}, \mathrm{y}$ there are disjoint open sets $\mathrm{a}, \mathrm{b} \in \mathrm{OX}$ such that $\mathrm{x} \in \mathrm{a}$ and $\mathrm{y} \in \mathrm{b}$.

(4.11) Theorem. A property system $\mathrm{P}$ satisfies the Leibnizian principle $\left(\mathrm{LPT}_{2}\right)$ iff for all distinct objects $\mathrm{x}$ and $\mathrm{y}$ there are properties $\mathrm{F}$ and $\mathrm{G}$ such that $\mathrm{x}$ has property $\mathrm{F}$ and all elements that have property $\mathrm{F}$ lack property $\mathrm{G}$, and y has property $\mathrm{G}$ and all elements that have g lack property $\mathrm{F}$. A topological space $(\mathrm{X}, \mathrm{OX})$ is a $\mathrm{T}_{2}$-space iff the topological property system $\{\mathrm{O}(\mathrm{x}) ; \mathrm{x} \in \mathrm{X}\}$ satisfies $\left(\mathrm{LPT}_{2}\right)$. 
The axioms $T_{0}, T_{1}$, and $T_{2}$ are not equivalent, but satisfy the strict chain of implications $\mathrm{T}_{2} \Rightarrow \mathrm{T}_{1} \Rightarrow \mathrm{T}_{0}$. Hence the principles $\left(\mathrm{LPT}_{0}\right),\left(\mathrm{LPT}_{1}\right)$ and $\left(\mathrm{LPT}_{2}\right)$ are not equivalent but satisfy the analogous chain of implication $\mathrm{LPT}_{2} \Rightarrow \mathrm{LPT}_{1} \Rightarrow \mathrm{LPT}_{0}$.

Separation axioms $\mathrm{T}_{\mathrm{i}}$ determine important features of topological spaces. It seems plausible to conjecture that the corresponding principles of identity $\left(\mathrm{LPT}_{\mathrm{i}}\right)$ determine important metaphysical features of the worlds for which they hold. Thus, topology turns out to provide useful means for distinguishing metaphysically different worlds in a more fine-grained way than the usual conceptual apparatus based on set theory. These examples may suffice to argue that topology is a conceptual useful device to define interesting and well-behaved property systems that give rise to non-trivial topological identity principles $\mathrm{LPT}_{\mathrm{i}}$. To be sure, there are other spatial structures that may can also be used for this purpose, for instance, convexity structures of conceptual spaces (see section 3). It should be noted, however, that convex structures and topological structures are structurally rather similar: Both can be defined by closure operators (cf. van de Vel (1993)).

5. Concluding Remarks. Not just any topological structure is appropriate as a means for distinguishing between good and not-so-good, i.e., natural and not-sonatural concepts. The point is to find illuminating topologies for specific purposes. This may be difficult: It may turn out that a certain (topological or convex) structure imposes formal conditions on naturalness that are unacceptable for intuitive reasons. If this is the case, then it has be replaced by another, more appropriate structure. Hopefully, thereby an equilibrium between our intuitions concerning naturalness and the formal conditions imposed by the spatial structures may be achieved. 
Since the end of 19th century a general topological concept of space has been developed that comprises the traditional Euclidean concept only as a very special case. Philosophy should attempt to exploit this newly gained general perspective. Then, topology as a general theory of spatial structures may serve as a kind of conceptual toolkit at least as versatile and fruitful as traditional logic. In terms of traditional philosophy of science the task of finding a "good" topology may be described as the task of formulating a kind of relativized or historized a priori element that is expressed in topological (= geometrical) terms. This thesis may be elucidated by briefly recalling an essential ingredient of Kant's original account that still plays an essential role in the contemporary discussion, namely, the a priori and its role in the constitution of the objects of science. For Kant, Euclidean geometry of space functioned as a constitutive framework for physics. This framework was not empirical - rather, it first rendered possible properly empirical discoveries (cf. Friedman $(2001,62))$. For example, Euclidean geometry enables us to represent physical phenomena such as the paths of particles, their velocities, and the forces that act on them geometrically or topologically, i.e., by certain spatial structures - for instance, by vectors, tensors, differential forms, and many other geometrical constructs.

Since Einstein's relativity theories one knows, however, that the framework of Euclidean geometry is not the only possible one. There is more than one constitutive geometrical framework possible, and these different frameworks compete with each other with respect to simplicity, fruitfulness, precision, and other theoretical and practical virtues. The different frameworks compete with each other. To take into account the dynamic aspects of the evolution of scientific knowledge, a particular 
structuralization of a conceptual space is to be conceived as only one phase in a process of the evolution of ever more sophisticated conceptual structures.

For the philosophical discussion concerning the role of a priori aspects of scientific knowledge, this means that pragmatic aspects of the a priori become increasingly important in post-Kantian philosophy of science (see sections 4 and 5).

The presence of spatial structures of some kind or other everywhere in the representation of empirical knowledge provides a good argument for the thesis that topology_may be a fruitful source for the formulation of a relativized or historized Kantian a priori. A detailed elaboration of this thesis is a task for future work.

\section{References.}

Aiello, M., Pratt-Hartmann, I.E., van Benthem, J.F.A.K., 2007, Handbook of Spatial Logics (eds.), Springer.

Albertazzi, L., 2000, Meaning and and Cognition. A multidisciplinary approach. (ed.), Amsterdam/Philadelphia, John Benjamins.

Cassirer, E., 1910 (1923), Substanzbegriff und Funktionsbegriff, translated as Substance and Function by W.C. Curtis and M.C. Curtis, Chicago, London, The Open Court Publishing Company.

Cassirer, E., 1928, Zur Theorie des Begriffs. Bemerkungen zu dem Aufsatz von Georg Heymans, Kant-Studien 33 (1-2), 129 - 136.

Douven, I., Gärdenfors, P., 2018, What are Natural Concepts? A Design Perspective, to appear in Mind and Language.

Forrest, P., 2016, The Identity of Indiscernibles, The Stanford Encyclopedia of Philosophy, Edward N. Zalta (ed.), URL $=<$ https://plato.stanford.edu/archives/win2016/entries/identity-indiscernible/>. 
Friedman, M., 2001, Dynamics of Reason, Stanford/California, CSLI Publications.

Gärdenfors, P., 2000, Conceptual Spaces. The Geometry of Thought, CambridgeMassachusetts, The MIT Press.

Gärdenfors, P., 2014, The Geometry of Meaning, Semantics based on Conceptual Spaces, Cambridge/Massachusetts, The MIT Press.

Heis, J., 2013, Ernst Cassirer, Kurt Lewin, and Hans Reichenbach, in N. Milkov, V. Peckhaus (eds.), The Berlin Group and the philosophy of Logical empiricism, Boston Studies in the History and Philosophy of Science 273, Springer, 67 - 94.

Johnstone, P.T., 1982, Stone Spaces, Cambridge, Cambridge University Press.

Lakoff, G., 1987, Women, Fire, and Dangerous Things. What Categories Reveal about the Mind, Chicago, University of Chicago Press.

Lakoff, G., Núñez, R., 2000, Where Mathematics Comes From. How the Embodied Mind Brings Mathematics into Being, New York, Basic Books.

Mac Lane, S., Moerdijk, I., 1992, Sheaves in Geometry and Logic. A First Introduction to Topos Theory, Springer.

Lewis, C.I., 1929 (1956), Mind and the World Order. Outline of a Theory of Knowledge, New York NY, Dover Publications.

Mormann, T., 1993, Natural Predicates and Topological Structures of Conceptual Spaces, Synthese 95(2), $219-240$.

Piazza, M., 1995, “One must always topologize”, Il teorema di Stone, la “ topologia influente" e l'epistemologia matematica, Rivista di storia della scienza (ser. II) 4, 1 24.

Peruzzi, A., 2000, The Geometric Roots of Semantics, in L. Albertazzi (ed.), 169 202.

Petitot, J., 1995, Morphodynamics and Attractor Syntax: Constituency in Visual 
Pattern Perception and Cognitive Grammar, in R.F. Port, T. Van Geldern (eds.), Mind as Motion, MIT Press, Cambridge/Massachusetts, $224-281$.

Rumfitt, I., 2015, The Boundary Stones of Logic. An Essay in the Philosophy of Logic, Oxford, Oxford University Press.

Steen, L.A., Seebach Jr., J.A., 1978, Counterexamples in Topology, Springer.

Stone, M.H., 1936, The Theory of Representations for Boolean Algebras, Transactions of the American Mathematical Society 40, 36 - 111.

Stone, M.H., 1937, Applications of the Theory of Boolean Rings to General Topology, Transactions of the American Mathematical Society 41, 345 -481.

Wildgen, W., 1983, Modelling Vagueness in Catastrophe Theoretic Semantics, in Ballmer, T., Pinkal, M. (eds.) Approaching Vagueness, North-Holland, Amsterdam, $317-360$.

Wildgen, W. 2000, The History and Future of Field Semantics: From Giordano Bruno to Dynamic Semantics, in L. Albertazzi (ed.), 203 - 226.

Wildgen, W., 2001, Kurt Lewin and the Rise of "Cognitive Science" in Germany: Cassirer, Bühler, Reichenbach, in Albertazzi, L. (ed.), The Dawn of Cognitive Science. Early European Contributions, Series Synthese, Kluwer, Dordrecht, 299 332.

Van de Vel, M.L.J., 1993, Theory of Convex Structures, Amsterdam, North-Holland. Zenker, F., Gärdenfors, P., 2015, Applications of Conceptual Spaces. The Case of Geometric Knowledge Representation (eds.), Synthese Library 359, Springer. 